\title{
ON THE POLYGONAL CONNECTIVITY OF POLYHEDRA AND THE CLOSURES OF OPEN CONNECTED SETS
}

\author{
BY NICK M. STAVRAKAS \\ Communicated by Everett Pitcher, September 27, 1972
}

\begin{abstract}
Generalizations of Tietze's theorem on convex sets are given in restricted settings. For example Theorem: Let $S \subset R^{d}$ be a connected polyhedron locally homeomorphic to the closed $d$ ball. If the points of local nonconvexity of $S$ are decomposable into $n$ convex sets, then $S$ is $n+1$ polygonally connected.
\end{abstract}

1. Introduction. F. A. Valentine in [6] proves the following results.

THEOREM 1. Let $S$ be a closed connected subset of $R^{d}$ which has at most $n$ points of local nonconvexity. Then $S$ is $n+1$ polygonally connected.

THEOREM 2. Let $S$ be a closed connected subset of $R^{d}$. Suppose that the points of local nonconvexity of $S$ are decomposable into $n$ convex sets. Then $S$ is $2 n+1$ polygonally connected.

These results have been extended by a number of authors, but always with stronger hypothesis. See [1] and [3]. Utilizing a minimal are technique, new proofs of Theorems 1 and 2 are given in [4], and a new characterization of the convex kernel in [5]. Guay and Kay in [2] give a new proof of Tietze's theorem using minimal arcs and the concept of $m$-convexity.

Valentine in [6] poses the problems of improving Theorem 2 in the cases where $S$ is the closure of open connected set in $R^{d}$ or a polyhedron in $R^{d}$. The purpose of the present paper is to announce such improvements for particular types of polyhedra and open connected sets.

2. The improvement for the closures of open connected sets. If $A$ is a set, $\bar{A}$ and $L(A)$ denote the closure of $A$ and the points of local nonconvexity of $A$, respectively.

DeFinition 1. Let $S$ be an open connected subset of $R^{d}$. We say $S$ is locally convex connected provided, given $x \in \bar{S}$ and an open set $N_{x}^{*}$ about $x$, there exists an open convex set $N_{x}$ about $x$ such that

1. $N_{x} \subset N_{x}^{*}$,

2. $N_{x} \cap S$ is connected,

3. $L\left(\overline{\left.N_{x} \cap S\right)}=\bar{N}_{x} \cap L(\bar{S})\right.$.

Our improvement is the following theorem.

THEOREM 3. Let $S$ be an open connected subset of $R^{d}$ which is locally

AMS (MOS) subject classifications (1970). Primary 52A30; Secondary 52A20. 
convex connected. Suppose the points of local nonconvexity of $S$ are decomposable into $n$ closed convex sets $C_{i}, 1 \leqq i \leqq n$, where $\operatorname{dim} C_{i} \leqq d-2$. Then $\bar{S}$ is $n+1$ polygonally connected.

To indicate a proof of Theorem 3, we develop the following notation. Let $S$ be a set satisfying the hypothesis of Theorem 3. The symbol $C(\bar{S})$ denotes the points of local convexity of $\bar{S}$. A point $z \in L(\bar{S})$ is called a simple point provided $z$ is contained in exactly one $C_{i}$. If $z$ is contained in two of the $C_{i}$ it is called a junction point. Let $x$ and $y$ be any two points in $\bar{S}$. Let $l$ be an arc from $x$ to $y$ in $\bar{S}$ of smallest arc length. It is well known that $l$ is a polygonal arc. Let $[a b]$ be a line segment contained in $l$. We call $[a b]$ a single segment provided $(a b) \subset C(\bar{S})$ and $a, b \in L(\bar{S})$. We call $[a b]$ a double segment provided $[a b] \subset L(\bar{S})$ and there do not exist points $c$ and $d$ such that $[a b] \subset[c d] \subset L(\bar{S})$ with $[c d] \subset l$. Let the vertices of $l$ be $\left\{x_{0}, x_{1}, \ldots, x_{k}, x_{k+1}\right\}$, where $x_{0}=x$ and $x_{k+1}=y$. Consider an $x_{i}, 1 \leqq i \leqq k$. We call $x_{i}$ locally left single provided there exists a point $c \in\left[x_{i-1}, x_{i}\right)$ such that $\left[c, x_{i}\right]$ is a single segment. We call $x_{i}$ locally left double provided there exists a point $c \in\left[x_{i-1}, x_{i}\right)$ such that $\left[c, x_{i}\right]$ is a double segment. The concepts of locally right single and locally right double are defined in the obvious way.

The following result is proven by a sequence of three lemmas, each lemma essentially being an application of Tietze's theorem.

THEOREM 4. Let $S$ satisfy the hypothesis of Theorem 3 with $n=1$. Then $C_{1}$ is contained in the convex kernel of $\bar{S}$.

Then, using Theorem 4, one establishes the following result, which exhibits the behavior of minimal arcs in the closures of open sets which are locally convex connected.

THEOREM 5. Let $S$ satisfy the hypothesis of Theorem 3. Let $x, y \in \bar{S}$ and let $l$ be a minimal arc from $x$ to $y$ in $\bar{S}$. Let $c$ be a vertex of l, c distinct from $x$ and $y$. Suppose $c$ is contained in a single segment of $l$ and a double segment of $l$. Then $c$ is a junction point.

The next theorem is vital in the proof of Theorem 3.

THEOREM 6. Let $S$ be a set satisfying the hypothesis of Theorem 3. Let $x, y \in \bar{S}$ and let $l$ be a minimal arc from $x$ to $y$ in $\bar{S}$. Let the vertices of $l$ strictly between $x$ and $y$ be $\left\{x_{1}, \ldots, x_{k}\right\}$. Let $A=\left\{C_{1}, \ldots, C_{n}\right\}$. Then there exists a set $\left\{C_{j_{1}}, \ldots, C_{j_{k}}\right\}$ such that, for each $i, 1 \leqq i \leqq k, C_{j_{i}} \in A$, $x_{i} \in C_{j_{i}}$ and, for $i \neq m, C_{j_{i}} \neq C_{j_{m}}$.

ProOF (SKETCH). We proceed by induction. For $k=1$, the theorem is clearly true. So suppose the theorem holds for $k-1$ and we prove the theorem for $k$. Consider the minimal arc $l^{\prime}$ from $x$ to $x_{k}$ determined by 
$\left\{x, x_{1}, \ldots, x_{k}\right\}$. The inductive hypothesis implies that, for each $i$, $1 \leqq i \leqq k-1$, there exists $C_{j_{i}} \in A$ such that $x_{i} \in C_{j_{i}}$ and, for each $i \neq m$, $C_{j_{i}} \neq C_{j_{m}}$. Now consider $x_{k}$. We classify $x_{k}$ into three types.

1. $x_{k}$ is locally left single.

2. $x_{k}$ is locally left double and locally right double.

3. $x_{k}$ is locally left double and locally right single.

We consider two cases:

Case $1 . x_{k}$ is of type 1. Since $x_{k}$ is a vertex of the minimal arc $l$, it is a point of local nonconvexity. Let $x_{k} \in C_{r}$. The minimality of $l$ implies that $C_{r} \neq C_{j_{i}}, 1 \leqq i \leqq k-2$. Also $C_{r} \neq C_{j_{k-1}}$, since if $C_{r}=C_{j_{k-1}},\left[x_{k-1}, x_{k}\right]$ would be a double segment, a contradiction. Thus $\left\{C_{j_{1}}, \ldots, C_{j_{k-1}}, C_{r}\right\}$ is the required set.

Case 2. $x_{k}$ is of type 2 or type 3 . If $x_{k}$ is of type 2 it is clearly a junction point. If $x_{k}$ is of type 3 , Theorem 5 implies that $x_{k}$ is a junction point. Thus, in either instance, $x_{k}$ is a junction point. Let $x_{k} \in C_{r}$ and $x_{k} \in C_{q}$. The minimality of $l$ implies that $C_{r} \neq C_{j_{i}}$ and $C_{q} \neq C_{j_{i}}, 1 \leqq i \leqq k-2$. Choose one element of $\left\{C_{r}, C_{q}\right\}$ which is not equal to $C_{j_{k-1}}$. W.l.o.g. suppose it is $C_{r}$. Then note $\left\{C_{j_{1}}, \ldots, C_{j_{k-1}}, C_{r}\right\}$ is the required set.

Corollary 1. Let $S$ satisfy the hypothesis of Theorem 3. Let $x, y \in \bar{S}$ and let $l$ be a minimal arc from $x$ to $y$ in $\bar{S}$. Let the vertices of $l$ be $\left\{x, x_{1}, \ldots, x_{k}, y\right\}$. Then $k \leqq n$.

Proof. Suppose $k \geqq n+1$. Since there are only $n$ of the $C_{i}$ to choose from, this implies that the set guaranteed by Theorem 6 does not exist, a contradiction.

Note that Theorem 3 follows immediately from Corollary 1 .

3. The improvement for polyhedra in $R^{d}$. Using techniques similar to those given in $\$ 2$ it is possible to prove

THEOREM 7. Let $P \subset R^{d}$ be a connected polyhedron which is locally homeomorphic to the closed d-ball. Suppose $L(P)$ is decomposable into $n$ convex sets. Then $P$ is $n+1$ polygonally connected.

Guay and Kay [1] have shown that if $S$ is a closed connected set in a topological vector space with $L(S)$ equal to exactly $n$ points and $S \sim L(S)$ connected, then $S$ is a union of $n+1$ or fewer closed convex sets. In the vein of this result we are able to show the following generalization of Tietze's theorem in $R^{3}$.

TheOREM 8. Let $S \subset R^{3}, S$ closed and $S$ not planar. Suppose $L(S)$ is decomposable into $n$ closed line segments. Suppose $S \subset \overline{C(S)}, C(S)$ is connected and given $x, y \in C(S)$ that they may be joined arc on arc $l \subset S$ such that $l$ is contained in a hyperplane. Then $S$ is $n+1$ polygonally connected. 


\section{BIBLIOGRAPHY}

1. M. Guay and D. Kay, On sets having finitely many points of local nonconvexity and property $P_{m}$, Israel J. Math. 10 (1971), 196-209.

2. $\longrightarrow$, Convexity and a certain property $P_{m}$, Israel J. Math. 8 (1970), 39-52.

3. N. M. Stavrakas, W. R. Hare and J. W. Kenelly, Two cells with n points of local nonconvexity, Proc. Amer. Math. Soc. 27 (1971), 331-336.

4. N. M. Stravrakas and R. E. Jamison, Valentine's extensions of Tietze's Theorem on convex sets, Proc. Amer. Math. Soc. (to appear)

5. N. M. Stravakas, The dimension of the convex kernel and points of local nonconvexity, Proc. Amer. Math. Soc. 34 (1972), 222-224.

6. F. A. Valentine, Local convexity and $L_{n}$ sets, Proc. Amer. Math. Soc. 16 (1965), 13051310. MR 32 \# 2976.

Department of Mathematics, Clemson University, Clemson, South Carolina 29631

Current address: Department of Mathematics, University of North Carolina, Raleigh, North Carolina 27607 